\title{
Coalition-proof supply function equilibria in oligopoly
}

\author{
Juan Delgado $^{\mathrm{a}, \mathrm{b}}$ and Diego Moreno ${ }^{\mathrm{b}, *}$ \\ ${ }^{a}$ European Commission, Competition Directorate-General (J70/2/110) Post, Telecommunications and \\ Information Society Co-ordination, B-1049 Brussels, Belgium \\ b Departmento de Economía, Universidad Carlos III de Madrid, 28903, Getafe, Spain
}

Received 6 March 2001; final version received 12 November 2002

\begin{abstract}
In an industry where firms compete via supply functions, the set of equilibrium outcomes is large. If decreasing supply functions are ruled out, this set is reduced significantly, but remains large. Specifically, the set of prices that can be sustained by supply function equilibria is the interval between the competitive price and the Cournot price. In sharp contrast, when the number of firms is above a threshold we identify (e.g., three if demand is linear), only the Cournot outcome can be sustained by a coalition-proof supply function equilibrium.
\end{abstract}

(C) 2003 Elsevier Science (USA). All rights reserved.

JEL classification: C72; D43; L13; L51

Keywords: Oligopoly; Cournot; Competition via supply functions; Coalition-proofness

\section{Introduction}

We study an oligopoly where firms compete via supply functions. In this setting, each firm chooses a supply function. The supply functions of all firms are then aggregated to form the market supply which, together with the market demand, determine the market clearing price and the production of each firm. This model of competition describes many markets more realistically than the Cournot or the Bertrand models. In markets for government procurement contracts, or in the

*Corresponding author. Fax: +34916249875.

E-mail addresses: juan.delgado-urdanibia@cee.eu.int (J. Delgado),dmoreno@eco.uc3m.es

(D. Moreno). 
recently deregulated electricity markets, for example, that the strategies of the firms are supply functions is a feature of the institutional setting.

A shortcoming of this model is that the set of outcomes that can be sustained by supply function equilibria (SFE henceforth) is very large see, e.g., Grossman [7], Hart [8]. Klemperer and Meyer [10] have studied the consequences of introducing demand uncertainty into the model, and have shown that if uncertainty is unbounded there is a unique equilibrium. Unfortunately, in many markets demand uncertainty plays a small role.

We study a version of the model where the strategies of the firms are restricted to non decreasing supply functions. This restriction seems reasonable and may add realism to the model: in many markets firms may not be able to commit to a decreasing supply schedule; in others, decreasing supply functions are explicitly ruled out by design. It turns out that restricting the strategy spaces significantly reduces the set of equilibria the monopoly price, for example, can no longer be sustained by SFE. Nevertheless, the set of equilibrium outcomes remains large. Specifically, the set of prices that can be sustained by SFE is the interval between the competitive price and the Cournot price. Further, the prices in this interval can be sustained by SFE leading to symmetric outcomes as well as by SFE leading to asymmetric ones. (The Cournot price is the unique exception: every SFE leading to this price yields the Cournot outcome.)

Next, we consider a setting where firms can communicate prior to taking an action, but cannot make binding commitments, and we study whether accounting for the opportunities of coordinated action that communication brings about provides a basis for determining which outcomes may arise. In non cooperative environments where players can communicate, equilibria are not viable unless they are invulnerable to improving deviations by any coalition of players. In other words, viable equilibria must be "coalition proof". A number of papers in the literature have studied which equilibria are coalition proof in a variety of oligopolistic environments under either Bertrand or Cournot competition see, e.g., [4,13]. We study the set of outcomes that can be sustained by coalition proof equilibria in our framework, and we find that the multiplicity of equilibrium outcomes disappears.

We introduce the notion of coalition proof supply function equilibrium (CPSFE henceforth), which is just an adaptation to our framework of Bernheim et al. [3] notion of coalition proof Nash equilibrium. A CPSFE is a profile of supply functions that is invulnerable to improving and self enforcing deviations by any coalition of firms. A deviation is self enforcing if there is no further self enforcing improving deviation available to a proper subcoalition of the deviating coalition. Note that deviations which are not self enforcing are meaningless as they are vulnerable to further self enforcing improving deviations by proper subcoalitions. (Aumann [1] introduced an alternative notion of coalition proof equilibrium, strong Nash equilibrium, which requires that a strategy profile be invulnerable to all improving coalitional deviations, self enforcing or not. This notion of equilibrium is so strong, however, that many games have no such equilibria.)

Since there are games for which a coalition proof Nash equilibrium does not exist (see [3]), we begin by studying whether the existence of a CPSFE is guaranteed in our 
framework. Indeed, we show that the Cournot outcome can be sustained by a CPSFE, and hence that in our framework a CPSFE always exists. Further, we identify conditions on the curvature of the demand and cost functions that guarantee that if the number of firms is above a threshold, the Cournot outcome is the unique CPSFE outcome. (If the market demand is a linear function, this result arises when there are three or more firms in the industry.)

Our proofs of unicity of a CPSFE outcome are based purely on Pareto dominance arguments: It turns out that when the number of firms in the industry is above a threshold, the Cournot outcome is Pareto superior to every other SFE outcome; i.e., the profits of every firm at the Cournot outcome are greater than at every other SFE outcome. Thus, since the Cournot outcome can be sustained by a CPSFE, every SFE leading to an outcome other than the Cournot outcome is upset by a self enforcing deviation of the grand coalition leading to the Cournot outcome.

In proving that the Cournot outcome is Pareto superior to every other SFE outcome, the class of SFE in which all but perhaps one firm supply inelastically the same fixed amount, and the remaining firm maximizes on the residual demand, plays an important role. (The set of outcomes sustained by SFE in this class can be easily characterized.) We show that for every SFE, there is a SFE in this class such that the profits (and outputs) of the firms using an inelastic supply function are greater or equal to those of the firm with the greatest profit (and output) in the original SFE. (This result indirectly establishes that SFE outcomes cannot be very asymmetric.) It turns out that if the number of firms is above a threshold, then the Cournot outcome (which can be sustained by a SFE in this class) is Pareto superior to every other outcome sustained by SFE in this class, and is therefore Pareto superior to every other SFE outcome.

It is worth noting that in the general context of non cooperative games coalition proof Nash equilibrium outcomes need not be Pareto optimal within the class of equilibrium outcomes. In our framework, however, the unique CPSFE outcome (i.e., the Cournot outcome) is the unique Pareto optimal outcome within the class of SFE outcomes. As a consequence, every SFE that leads to an outcome other than the Cournot outcome is vulnerable to a self enforcing deviation of the grand coalition, and is therefore very "unstable." This provides additional support for the Cournot outcome, since every SFE that leads to a different outcome is easily upset.

An interesting interpretation of our unicity result, parallel to Kreps and Scheinkman [11], is that the Cournot model provides a "reduced form" of a "structural model" where firms compete by choosing a supply function, but can communicate prior to taking an action. As we show, when the coordination opportunities brought about by the possibility of communication are taken into account, the Cournot model yields predictions identical to those obtained using the more cumbersome model of competition via supply functions. (See also [9] on this debate.)

\section{Supply function equilibria}

In this section we describe the model of competition via supply functions, we characterize the prices that can be sustained by supply function equilibria, and we 
show that every SFE leading to the Cournot price yields the Cournot outcome. Further, we show that within the set of SFE outcomes the profit of the industry is uniquely maximized at the Cournot outcome.

The description of the industry, except for allowing more than two firms, is identical to that of Klemperer and Meyer [10], and is common in the literature see, e.g., $[4,6,11]$. As in these papers, we assume that the demand function is concave and the cost function is convex. Also we assume, as in [10], that firms' supply functions are continuously differentiable.

These assumptions render tractable a complex model. (Note that since strategies are functions, the analysis of the game describing the industry is difficult.) Specifically, under these assumptions equilibrium outcomes are solutions to the system of equations formed by first order conditions for profit maximization and the market clearing condition. (In a SFE every firm maximizes profits on the "residual" demand, effectively determining the market clearing price. Thus, if every firm chooses a convex supply function, then under the above assumptions the profit of each firm is a concave and differentiable function of the market price.) Of course, these assumptions also simplify the analysis; they imply, for example, that the Cournot equilibrium is unique and symmetric, and that the profit of the industry increases with prices below the monopoly price. These results hold, however, under weaker assumptions.

In contrast to Klemperer and Meyer [10], we restrict the supply functions that firms can use to be non decreasing. This restriction is appropriate for the analysis of industries where firms cannot commit to a decreasing supply schedule, or to those where decreasing supply functions are ruled out by design, as in many organized markets. In the Spanish electricity market, for example, decreasing supply functions are explicitly ruled out see [5, 4.3.1 Simple Bids, p. 9, 4.6.1.6 Verification of the Contents of Simple Bids, p. 14]. It turns out that restricting supply functions to be non decreasing significantly reduces the set of prices that can be sustained by SFE. The monopoly price, in particular, can no longer be sustained by SFE the largest price that can be sustained by SFE is the Cournot price.

As in the papers mentioned above, symmetry plays an important role in the analysis. It seems, however, that appropriate versions of Proposition 2.1 and Theorem 2.2 hold for asymmetric industries, although the proofs will be considerably more cumbersome.

\subsection{The industry}

Demand is known to all firms with certainty. Throughout it is assumed that the demand function $D: \mathbb{R}_{+} \rightarrow \mathbb{R}_{+}$is twice continuously differentiable, strictly decreas ing, and concave on $(0, \rho)$, where $\rho>0$ satisfies $D(p)>0$ for $p<\rho$, and $D(p)=0$ for $p \geqslant \rho$. All firms have access to the same technology, and therefore have identical cost function, $C: \mathbb{R} \rightarrow \mathbb{R}$; we assume that $C$ is twice continuously differentiable, non decreasing and convex on $\mathbb{R}_{+}$, and satisfies $C^{\prime}(0)<\rho$, and $C(q)=C(0)$ for $q<0$. (Extending the domain of the cost function to include negative quantities is inconsequential and simplifies our analysis.) 
An industry is therefore described by a triple $(D, C, N)$, indicating the market demand, $D$, the cost function, $C$, and the set of firms, $N=\{1, \ldots, n\}$ where $n \geqslant 2$. In what follows, let us be given an industry $(D, C, N)$.

\subsection{Competition via supply functions}

Firms compete by simultaneously choosing a supply function, i.e., a non decreasing real valued function on $[0, \rho]$ that is continuously differentiable on $(0, \rho)$. For a profile of supply functions $s=\left(s_{1}, \ldots, s_{n}\right)$, a market clearing price is a solution to the equation

$$
\sum_{i=1}^{n} s_{i}(p)=D(p)
$$

Since the market demand is strictly decreasing and the supply function of each firm is non decreasing, if a market clearing price exists, then it is unique. For each profile of supply functions $s$, let $p(s)$ be the market clearing price if it exists, and let $p(s)$ be zero if a market clearing price does not exist. The profit (payoff) of a firm $i \in N$ is given by $\pi_{i}(s)=p(s) s_{i}(p(s)) \quad C\left(s_{i}(p(s))\right)$. (This construction implicitly assumes that when a market clearing price does not exist the revenue of every firm is zero.)

A supply function equilibrium (SFE) is a (pure strategy) Nash equilibrium of the game described. Write $\operatorname{SFE}(D, C, N)$ for the set of supply function equilibria. In a SFE each firm maximizes profits on its "residual demand"; that is, if $\hat{s} \in \operatorname{SFE}(D, C, N)$, then $p(\hat{s})=\hat{p}$ solves

$$
\max _{p \in[0, \rho]} p\left(D(p) \sum_{j \neq i} \hat{s}_{j}(p)\right) \quad C\left(D(p) \sum_{j \neq i} \hat{s}_{j}(p)\right),
$$

for each $i \in N$. If $\hat{p} \in(0, \rho)$, then $\hat{s}$ satisfies

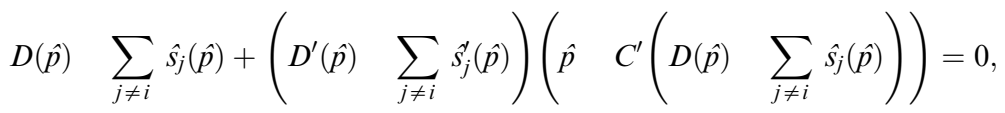

for each $i \in N$. Writing $\hat{q}_{i}=\hat{s}_{i}(\hat{p})$, and using the market clearing condition (MC), this condition can be written for each $i \in N$ as

$$
\hat{q}_{i}+\left(D^{\prime}(\hat{p}) \quad \sum_{j \neq i} \hat{s}_{j}^{\prime}(\hat{p})\right)\left(\hat{p} \quad C^{\prime}\left(\hat{q}_{i}\right)\right)=0 .
$$

Because the demand function is concave and the cost function is convex, if in addition each $\hat{s}_{i}$ is a convex function, then satisfying the system of equations formed by $\left(\mathrm{E}_{1}\right), \ldots,\left(\mathrm{E}_{n}\right)$ and $(\mathrm{MC})$ is a sufficient condition for a strategy profile $\hat{s}$ to be a SFE. Thus, every equilibrium outcome $\left(p, q_{1}, \ldots, q_{n}\right)$ such that $0<p<\rho$ must be a solution to the system of equations formed by $\left(E_{1}\right), \ldots,\left(E_{n}\right)$, and $(\mathrm{MC})$, for some profile of supply functions $s$. For $s \in \operatorname{SFE}(D, C, N)$, we denote by $\left(p(s), q_{1}(s), \ldots, q_{n}(s)\right)$ the associated equilibrium outcome. 


\subsection{Competitive and Cournot prices}

In determining the market prices that can be sustained by SFE the competitive price and the Cournot price play an important role. In our framework each of these prices is a solution to a system of equations.

A Cournot outcome $\left(\bar{p}, \bar{q}_{1}, \ldots, \bar{q}_{n}\right)$ is characterized by the system of equations

$$
\bar{q}_{i}+\left(\bar{p} \quad C^{\prime}\left(\bar{q}_{i}\right)\right) D^{\prime}(\bar{p})=0,
$$

for $i \in N$, and

$$
\sum_{i=1}^{n} \bar{q}_{i}=D(\bar{p})
$$

Eq. $(\overline{\mathrm{C}} 1)$ ensures that the output of each firm maximizes its profits given the aggregate output of its rivals, whereas $(\overline{\mathbf{C}} 2)$ ensures that the market clears.

Our assumptions on demand and cost functions imply the existence of a unique Cournot outcome, which is symmetric (i.e., satisfies $\bar{q}_{1}=\cdots=\bar{q}_{n}$ ). (The Cournot equilibrium is unique and symmetric under much weaker assumptions on the curvature of demand and cost functions; see, e.g., [2].) Given an industry $(D, C, N)$, we denote by $\bar{p}(D, C, N)$ and $\bar{q}(D, C, N)$ the market price and the output of each firm, respectively, at the Cournot outcome.

A competitive equilibrium $\left(\underline{p}, \underline{q}_{1}, \ldots, \underline{q}_{n}\right)$ satisfies the system of equations

$$
C^{\prime}\left(\underline{q}_{i}\right)=\underline{p},
$$

for $i \in N$, and

$$
\sum_{i=1}^{n} \underline{q}_{i}=D(\underline{p})
$$

Our assumptions on demand and cost functions imply the existence of a unique competitive price, which we denote by $\underline{p}(D, C, N)$. Clearly

$$
0 \leqslant \underline{p}(D, C, N)<\bar{p}(D, C, N)<\rho .
$$

\subsection{Results}

Proposition 2.1 shows that if $s$ is a SFE that leads to the Cournot price, then the outcome associated with $s$ is the Cournot outcome. Moreover, in every SFE that sustains the Cournot outcome, the derivative of the supply function of every firm vanishes at the Cournot price.

Proposition 2.1. Let $(D, C, N)$ be an industry, and let $s \in \operatorname{SFE}(D, C, N)$. If $p(s)=$ $\bar{p}(D, C, N)$, then $s_{i}(\bar{p})=\bar{q}(D, C, N)$, and $s_{i}^{\prime}(\bar{p})=0$ for $i \in N$.

Proof. Let $s \in \operatorname{SFE}(D, C, N)$ be such that $p(s)=\bar{p}(D, C, N)=\bar{p}$. Since $\bar{p} \in(0, \rho), s$ satisfies condition $\left(\mathrm{E}_{i}\right)$ for $i \in N$. We show that $s_{i}(\bar{p})=\bar{q}(D, C, N)=\bar{q}$ for $i \in N$. Assume by way of contradiction that there is a firm $j$ such that $s_{j}(\bar{p}) \neq \bar{q}$. Since 
$\sum_{i{ }_{1}}^{n} s_{i}(\bar{p})=D(\bar{p})=n \bar{q}$, assume without loss of generality that $s_{j}(\bar{p})=q_{j}<\bar{q}$. Then, because $D$ is decreasing, $C$ is convex, and each $s_{i}$ is non decreasing, we have

$$
\begin{aligned}
& 0=\bar{q}+\left(\bar{p} \quad C^{\prime}(\bar{q})\right) D^{\prime}(\bar{p})>q_{j}+\left(\bar{p} \quad C^{\prime}\left(q_{j}\right)\right) D^{\prime}(\bar{p})
\end{aligned}
$$

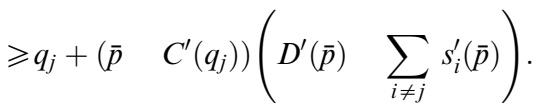

Thus, condition $\left(\mathrm{E}_{j}\right)$ is not satisfied, which is a contradiction.

We show that $s_{i}^{\prime}(\bar{p})=0$ for $i \in N$. For $i \in N$ and $j \in N \backslash i,\left(\mathrm{E}_{j}\right)$ and $\left(\overline{\mathrm{C}}_{1}\right)$ imply

$$
0=\bar{q}+D^{\prime}(\bar{p})\left(\bar{p} \quad C^{\prime}(\bar{q})\right)=\bar{q}+\left(D^{\prime}(\bar{p}) \quad \sum_{k \neq j} s_{k}^{\prime}(\bar{p})\right)\left(\bar{p} \quad C^{\prime}(\bar{q})\right) .
$$

Therefore $\sum_{k \neq j} s_{k}^{\prime}(\bar{p})=0$, and since $s_{k}^{\prime} \geqslant 0$ for $k \neq j$, we have $s_{i}^{\prime}(\bar{p})=0$.

Note that concavity of demand plays no role in the proof of Proposition 2.1.

Next, we study the set of outcomes that can be sustained by SFE. Klemperer and Meyer [10] have shown that when supply functions are not restricted to be non decreasing, then every outcome $\left(p, q_{1}, \ldots, q_{n}\right) \in \mathbb{R}_{+}^{n}$ such that $D(p)=\sum_{i 1}^{n} q_{i}$ and $C^{\prime}\left(q_{i}\right)<p$ for each $i \in N$ can be sustained by a SFE; in particular, the monopoly price can be sustained by SFE. Restricting supply functions to be non decreasing significantly reduces this set; in particular, the monopoly price can no longer be sustained as a SFE.

Write $\operatorname{SFE}_{p}(D, C, N)$ for the set of prices that can be sustained by SFE, i.e., $p \in \operatorname{SFE}_{p}(D, C, N)$ if $p=p(s)$ for some $s \in \operatorname{SFE}(D, C, N)$. Theorem 2.2 establishes that $\operatorname{SFE}_{p}(D, C, N)$ is the half open interval containing the prices between the competitive price and the Cournot price.

Theorem 2.2. $\operatorname{SFE}_{p}(D, C, N)=(\underline{p}(D, C, N), \bar{p}(D, C, N)]$.

Proof. Write $\underline{p}(D, C, N)=\underline{p}$ and $\bar{p}(D, C, N)=\bar{p}$. Let $\hat{p} \in(\underline{p}, \bar{p}]$; we show that there is $\hat{s} \in \operatorname{SFE}(D, C, N)$ such that $p(\hat{s})=\hat{p}$. Write $\hat{q}=\frac{D(\hat{p})}{n}$, and for $i \in N$ let $\hat{s}_{i}(p)=\hat{a}+\hat{\alpha} p$, where $\hat{a}$ and $\hat{\alpha}$ satisfy

$$
\hat{a}+\hat{\alpha} \hat{p}=\hat{q}
$$

and

$$
\hat{q}+\left(\hat{p} \quad C^{\prime}(\hat{q})\right)\left(D^{\prime}(\hat{p}) \quad(n \quad 1) \hat{\alpha}\right)=0 .
$$

Note that $\hat{s}_{i}^{\prime}(p)=\hat{\alpha}$ for $i \in N$. We show that $\hat{\alpha} \geqslant 0$, and therefore that each $\hat{s}_{i}$ is non decreasing. Since $\hat{p} \leqslant \bar{p}$ and $D$ is decreasing we have $\hat{q} \geqslant \bar{q}$, and since $D$ concave and $C$ is convex we have

$$
\hat{q}+\left(\hat{p} \quad C^{\prime}(\hat{q})\right) D^{\prime}(\hat{p}) \geqslant \bar{q}+\left(\bar{p} \quad C^{\prime}(\bar{q})\right) D^{\prime}(\bar{p})=0,
$$


and therefore

$$
\hat{\alpha}=\frac{1}{n \quad 1}\left(D^{\prime}(\hat{p})+\frac{\hat{q}}{\hat{p} \quad C^{\prime}(\hat{q})}\right) \geqslant 0 .
$$

Eq. (2.1) and the definition of $\hat{q}$ guarantee that $\hat{p}$ is the market clearing price for $\hat{s}=\left(\hat{s}_{1}, \ldots, \hat{s_{n}}\right)$. Eq. (2.2) ensures that $\hat{s}$ satisfies $E_{i}$ for $i \in N$. Since each $\hat{s_{i}}$ is convex, we have $\hat{s} \in \operatorname{SFE}(D, C, N)$.

Let $\hat{s}$ be such that $p(\hat{s})=\hat{p} \leqslant p<\rho$. We show that $\hat{s} \notin \operatorname{SFE}(D, C, N)$. Since $D$ is decreasing, we have $D(\hat{p}) \geqslant D(\underline{p})>D(\rho)=0$; hence there is one firm $i \in N$ producing $\hat{s}_{i}(\hat{p})=\hat{q}_{i} \geqslant \frac{D(p)}{n}>0$, and since $C$ is convex, $(\underline{C} 1)$ implies that $C^{\prime}\left(\hat{q}_{i}\right) \geqslant C^{\prime}\left(\frac{D(p)}{n}\right)=\underline{p} \geqslant \hat{p}$. Since $s_{j}^{\prime}(\hat{p}) \geqslant 0$ for $j \in N$, and $D$ is decreasing we have

$$
\hat{q}_{i}+\left(D^{\prime}(\hat{p}) \quad \sum_{j \neq i} \hat{s}_{j}^{\prime}(\hat{p})\right)\left(\hat{p} \quad C^{\prime}\left(\hat{q}_{i}\right)\right)>0 .
$$

Thus, condition $\left(\mathrm{E}_{i}\right)$ is not satisfied. Moreover, if $\hat{p}=0$ then we have $\pi_{i}(\hat{s})=$ $C\left(\hat{q}_{i}\right)<C(0)$. Hence $\hat{s} \notin \operatorname{SFE}(D, C, N)$.

Finally, let $\hat{s}$ be such that $\hat{p}>\bar{p}>0$. We show that $\hat{s} \notin \operatorname{SFE}(D, C, N)$. Since $D$ is strictly decreasing, we have $D(\hat{p})<D(\bar{p})$. Consequently, at least one firm $i \in N$ is producing $\hat{s}_{i}(\hat{p})=\hat{q}_{i}<\bar{q}=\bar{q}(D, C, N)$. Thus, since $\hat{s}_{i}^{\prime}(\hat{p}) \geqslant 0$, for $i \in N$, and $D^{\prime}(\hat{p}) \leqslant D^{\prime}(\bar{p})$ (recall that $D$ is concave), $(\overline{\mathrm{C}} 1)$ implies

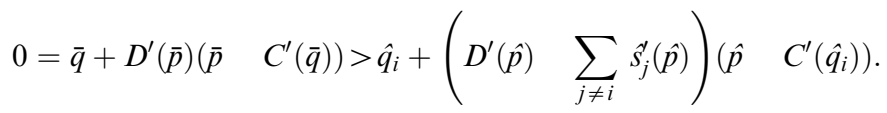

Hence condition $\left(\mathrm{E}_{i}\right)$ is not satisfied, and therefore $\hat{s} \notin \operatorname{SFE}(D, C, N)$.

Note that both concavity of demand and convexity of cost are used throughout the proof of Theorem 2.2. Also note that the construction in the proof of Theorem 2.2 establishes that every price between the competitive price and the Cournot price can be sustained by a symmetric SFE, whose associated outcome is therefore symmetric (i.e., all firms produce the same quantity). Nevertheless, there are asymmetric outcomes that can be sustained by SFE (see Example 3.6). It is also interesting to observe that in the SFE constructed in the proof of the Theorem 2.2, the parameter $\hat{\alpha}$ ranges from zero for $\hat{p}=\bar{p}$, to infinity as $\hat{p}$ approaches $\underline{p}$ (because $\hat{p} \quad C^{\prime}(\hat{q})$ approaches zero).

The following remark is a direct implication of Theorem 2.2.

Remark 2.3. For each $s \in \operatorname{SFE}(D, C, N)$, we have $p(s) \in(0, \rho)$. Hence every SFE outcome is a solution to the system of equations $\left(\mathrm{E}_{1}\right), \ldots,\left(\mathrm{E}_{n}\right),(\mathrm{MC})$, for some profile $s$ of supply functions.

We finish this section by showing in Proposition 2.4 that within the set of outcomes that can be sustained by SFE, the profit of the industry is uniquely 
maximized at the Cournot outcome. This result plays an important role in establishing in Section 3 that the Cournot outcome can be sustained by a CPSFE.

Proposition 2.4. Let $(D, C, N)$ be an industry, and let $\bar{s}, \hat{s} \in \operatorname{SFE}(D, C, N)$ be such that $p(\bar{s})=\bar{p}(D, C, N) \neq p(\hat{s})$. Then $\sum_{i 1}^{n} \pi_{i}(\bar{s})>\sum_{i 1}^{n} \pi_{i}(\hat{s})$.

Proof. Write $\bar{p}(D, C, N)=\bar{p}$, and for $p \in(0, \rho)$, define

$$
\Pi(p)=p D(p) \quad n C\left(\frac{D(p)}{n}\right) .
$$

Since $\Pi$ is concave (because $D$ and $C$ are concave), and

$$
\begin{aligned}
\Pi^{\prime}(\bar{p}) & =D(\bar{p})+D^{\prime}(\bar{p})\left(\begin{array}{ll}
\bar{p} & \left.C^{\prime}\left(\frac{D(\bar{p})}{n}\right)\right)
\end{array}\right) \\
& =D(\bar{p}) \quad \frac{D(\bar{p})}{n}=\frac{\left(\begin{array}{ll}
n & 1
\end{array}\right) D(\bar{p})}{n}>0,
\end{aligned}
$$

we have $\Pi^{\prime}(p)>0$ for $0<p \leqslant \bar{p}$. Therefore $\Pi(\bar{p})>\Pi(p)$ for $0<p<\bar{p}$.

Let $\bar{s}, \hat{s} \in \operatorname{SFE}(D, C, N)$ be such that $p(\bar{s})=\bar{p} \neq \hat{p}=p(\hat{s})$. Then $\bar{p}>\hat{p}$ by Theorem 2.2. Further, $q_{i}(\bar{s})=\bar{q}(D, C, N)=\frac{D(p)}{n}$ by Proposition 2.1, and since $C$ is convex, we have

$$
\sum_{i=1}^{n} \pi_{i}(\bar{s})=\Pi(\bar{p})>\Pi(\hat{p}) \geqslant \sum_{i=1}^{n}\left(\hat{p} q_{i}(\hat{s}) \quad C\left(q_{i}(\hat{s})\right)\right)=\sum_{i=1}^{n} \pi_{i}(\hat{s}),
$$

which establishes Proposition 2.4.

The following remark, which a direct implication of Proposition 2.4, establishes that the Cournot outcome is not Pareto dominated by any other SFE outcome.

Remark 2.5. Let $(D, C, N)$ be an industry, and let $\bar{s} \in \operatorname{SFE}(D, C, N)$ be such that $p(\bar{s})=\bar{p}(D, C, N)$. Then there is no $\tilde{s} \in \operatorname{SFE}(D, C, N)$ satisfying $\pi_{i}(\tilde{s})>\pi_{i}(\bar{s})$ for all $i \in N$.

\section{Coalition-proof supply function equilibria}

In this section we introduce the notion of coalition proof supply function equilibrium (CPSFE), and show that in our framework a CPSFE exists. Further, under appropriate conditions there is a unique CPSFE outcome. Remarkably, this outcome is the Cournot outcome. In both existence and uniqueness of CPSFE outcomes, restricting the supply functions of firms to be non decreasing plays an essential role. We comment on this issue below. We also discuss in Section 3.4 the robustness of the uniqueness result to cost asymmetries. 


\subsection{The notion of CPSFE}

In environments where players can freely discuss their strategies but cannot make binding commitments, viable agreements must be invulnerable to improving deviations by either individual players or coalitions of players. Thus, in these environments it is natural to ask which equilibria are "coalition proof." Indeed, this question has been studied in a variety of contexts, including political economy, social choice, and industrial organization e.g., Bernheim and Whinston [4], and Yi [13] study this issue in a variety of oligopolistic environments.

Several alternative notions of coalition proof equilibrium have been proposed in the literature. Aumann [1] introduced the notion of strong Nash equilibrium, which requires that a strategy profile be invulnerable to all improving coalitional deviations. The notion of strong Nash equilibrium is often too strong. Many games fail to have equilibria of this kind: in a Prisoner's Dilemma game, for example, the unique Nash equilibrium is not strong because it is vulnerable to the deviation where both players choose the cooperative action; note, however, that this deviation is meaningless as it is itself vulnerable to a further improving deviation by either player.

We formalize the notion of coalition proof supply function equilibrium (CPSFE henceforth), which is just an adaptation to our framework of the notion of coalition proof Nash equilibrium introduced by Bernheim et al. [3]. A CPSFE is a profile of supply functions that is invulnerable to improving and self enforcing deviations by any coalition of firms. A deviation is self enforcing if there is no further self enforcing improving deviation available to a proper subcoalition of the deviating coalition. Thus, following Bernheim et al. [3] we do not require that a profile of supply functions be invulnerable to all deviations, but just to those that are invulnerable to further self enforcing deviations by proper subcoalitions. We begin by formally defining the notion of CPSFE.

Let $(D, C, N)$ be an industry. We denote by $2^{N}$ the set of all possible coalitions. For a strategy profile $s$ and a coalition $M \in 2^{N}$, write $s_{M}$ for the profile of supply functions of the members of $M$, and write $m$ for the cardinality of the set $M$. Let $s$ be a strategy profile and let $M \in 2^{N}, 2 \leqslant m<n$, be a coalition of firms (recall that $n \geqslant 2$ ). Holding fixed the strategies of the members of the complementary coalition, $s_{N \backslash M}$, the situation the group of firms in $M$ faces can be modeled as that of an "industry" $\left(D_{s, M}, C, M\right)$, where $D_{s, M}$ is given for $p \in \mathbb{R}_{+}$by

$$
D_{s, M}(p)= \begin{cases}D(p) \quad \sum_{i \in N \backslash M} s_{i}(p) & \text { if } D(p) \quad \sum_{i \in N \backslash M} s_{i}(p) \geqslant 0 \\ 0 & \text { otherwise. }\end{cases}
$$

This recursive structure allows us to formalize the notion of CPSFE.

Coalition proof supply function equilibrium: Let $(D, C, N)$ be an industry.

(1) If $n=2$, a strategy profile $s$ is a coalition proof supply function equilibrium if $s \in \operatorname{SFE}(D, C, N)$ and there is no $\tilde{s} \in \operatorname{SFE}(D, C, N)$ satisfying $\pi_{i}(\tilde{s})>\pi_{i}(s)$ for every $i \in N$. 
(2) Let $n>2$ and assume that the notion of coalition proof supply function equilibrium has been defined for industries with fewer than $n$ firms.

(i) A strategy profile $s$ is self enforcing if $s \in \operatorname{SFE}(D, C, N)$, and if for all $M \in 2^{N}$, $2 \leqslant m<n, s_{M}$ is a coalition proof supply function equilibrium of the industry $\left(D_{S, M}, C, M\right)$.

(ii) A strategy profile $s$ is a coalition proof supply function equilibrium if it is self enforcing and if there is no self enforcing strategy profile $\tilde{s}$ such that $\pi_{i}(\tilde{s})>\pi_{i}(s)$ for every $i \in N$.

The definition of CPSFE applies to industries with no fewer than two firms. Note that a CPSFE is a SFE, and therefore it is invulnerable to deviations by a single firm. Given an industry $(D, C, N)$, write $\operatorname{CPSFE}(D, C, N)$ for the set of supply function equilibria.

\subsection{Existence of CPSFE}

Since there are no general results on the existence of coalition proof Nash equilibrium, we begin by establishing in Theorem 3.1 that every industry has a CPSFE. (Moreno and Wooders [12] provide conditions on the set of iteratively undominated strategies that guarantee existence of a coalition proof Nash equilibrium. In our framework, iterated elimination of strictly dominated strategies will not take us very far.)

Theorem 3.1. Every industry has a coalition proof supply function equilibrium.

Theorem 3.1 is a direct implication of Proposition 3.2 below, which establishes that the Cournot outcome can be sustained by a CPSFE. The proof of Proposition 3.2 relies on the recursive structure of Cournot equilibrium. The argument of the proof is analogous to that used by Bernheim and Whinston [4] to establish that in an industry (as the one described here) where firms compete à la Cournot, the unique equilibrium is a coalition proof Nash equilibrium. Since in our context we have multiplicity of equilibria, our proof relies on the fact that the Cournot outcome is on the Pareto frontier of the set of SFE outcomes (see Remark 2.5). Thus, restricting the supply functions of firms to be non decreasing plays an essential role in this argument. Indeed, if this restriction is removed, then the monopoly outcome where firms share the market equally arises as a SFE outcome see [10] and therefore the Cournot outcome is no longer on the Pareto frontier of the set of SFE outcomes.

Proposition 3.2. The Cournot outcome can be sustained by a CPSFE. Moreover, every SFE that yields the Cournot outcome is a CPSFE.

Proof. Let $(D, C, N)$ be an industry, and let $s \in \operatorname{SFE}(D, C, N)$ be such that $p(s)=$ $\bar{p}(D, C, N)=\bar{p}$ and $q_{i}(s)=\bar{q}(D, C, N)=\bar{q}$ for $i \in N$. (The existence of a SFE leading to the Cournot outcome is guaranteed by Theorem 2.2 and Proposition 2.1.) We show by induction on the number of firms that $s \in \operatorname{CPSFE}(D, C, N)$. 
If $n=2$, then $s \in \operatorname{CPSFE}(D, C, N)$ follows from Remark 2.5. Let $n>2$, and assume that $s \in \operatorname{CPSFE}(D, C, K)$ whenever $2 \leqslant k<n$. We show that $s \in \operatorname{CPSFE}(D, C, N)$. Note that for $M \in 2^{N}$ such that $2 \leqslant m<k$ the Cournot outcome of the industry $\left(D_{s, M}, C, M\right)$ is also $(\bar{p}, \bar{q}, \ldots, \bar{q})$; hence the induction hypothesis implies $s_{M} \in \operatorname{CPSFE}\left(D_{s, M}, C, M\right)$. Since $s \in \operatorname{SFE}(D, C, N), s$ is self enforcing. Moreover, since any other self enforcing strategy $\tilde{s}$ is a member of $\operatorname{SFE}(D, C, N)$, $s \in \operatorname{CPSFE}(D, C, N)$ follows from Remark 2.5.

\subsection{Uniqueness of CPSFE outcomes}

Next, we establish conditions under which the Cournot outcome is the unique CPSFE outcome. We show that when the number of firms in the industry is above a threshold, the Cournot outcome is Pareto superior to every other SFE outcome (i.e., the profits of every firm at the Cournot outcome are greater than at every other SFE outcome). Thus, any CPSFE leading to the Cournot outcome, which exist by Proposition 3.2, is a self enforcing and improving deviation of the grand coalition to every SFE leading to an outcome other than the Cournot outcome. Hence the Cournot outcome is the unique CPSFE outcome.

In proving that the Cournot outcome is Pareto superior to every other SFE outcome, a particular class of SFE plays an important role. In this class all but perhaps one firm supply inelastically the same fixed amount, and the remaining firm maximizes on the residual demand. (The Cournot outcome, in particular, can be sustained by a SFE in this class.) The SFE outcomes in this class are solutions to a simple system of equations, and can be parametrized by the slope of the supply function of the firm maximizing on the residual demand, evaluated at the market clearing price.

We show that for every SFE, $\hat{s}$, one can find a SFE in this class, $\tilde{s}$, for which the profits of the firms using an inelastic supply function (say firms 1 to $n$ 1) are greater or equal to those obtained by the firm with the greatest profits in the original SFE (i.e., $\left.\pi_{1}(\tilde{s})=\cdots=\pi_{n-1}(\tilde{s}) \geqslant \max \left\{\pi_{1}(\hat{s}), \ldots, \pi_{n}(\hat{s})\right\}\right)$. This key result is established in Proposition 3.3. As a direct consequence of this result, when the Cournot outcome is Pareto superior to every other outcome in this class, it is in fact Pareto superior to every other SFE outcome, and is therefore the unique CPSFE outcome (Proposition 3.4).

Let $(D, C, N)$ be an industry. Consider the outcomes that can be sustained by $s \in \operatorname{SFE}(D, C, N)$ for which there is $j \in N$ such that $s_{i}(p)=v \in \mathbb{R}_{+}$for each $i \in N \backslash\{j\}$ and $p \in[0, \rho]$. Writing $p(s)=u$, and $s_{j}(u)=w$, we can obtain these outcomes $(u, v, w) \in \mathbb{R}_{+}^{3}$ as solutions to a system of equations $(\mathrm{E}(\alpha))$ given by

$$
\begin{aligned}
& w=D^{\prime}(u)\left(u \quad C^{\prime}(w)\right), \\
& v=\left(\begin{array}{ll}
\alpha & \left.D^{\prime}(u)\right)\left(\begin{array}{ll}
u & C^{\prime}(v)
\end{array}\right), \\
(n \quad 1) v+w=D(u),
\end{array}\right.
\end{aligned}
$$


for $\alpha \in \mathbb{R}_{+}$. (In system $(E(\alpha))$, the parameter $\alpha$ is the slope of the supply function of firm $j$, the firm using an elastic supply, evaluated at the market clearing price.) System $(\mathrm{E}(\alpha))$ implicitly defines a function $(u(\alpha), v(\alpha), w(\alpha))$ on $[0, \infty)$ that is continuously differentiable on $(0, \infty)$. (In the proof of Proposition 3.3, given in the appendix, we show that fixing the production of a firm with the greatest production in an arbitrary SFE, $v \in \mathbb{R}_{+}$, system $(\mathrm{E}(\alpha))$ has a unique solution, $(u(v), w(v), \alpha(v))$. Moreover, $\alpha^{\prime}>0$, and therefore the function $\alpha$ is invertible.) Define the function $\pi$ on $[0, \infty)$ by

$$
\pi(\alpha)=u(\alpha) v(\alpha) \quad C(v(\alpha)) .
$$

Note that $\pi$ is continuously differentiable on $(0, \infty)$.

For $\alpha \geqslant 0$ denote by $\operatorname{SFE}_{\alpha}(D, C, N)$ the set of SFE of the form described above. Every $s \in S_{F E}(D, C, N)$ leads to an outcome given by $p(s)=u(\alpha), q_{i}(s)=v(\alpha)$ for $i \in N \backslash\{j\}$, and $q_{j}(s)=w(\alpha)$. The function $\pi(\alpha)$ provides the profits of the firms using an inelastic supply. Note that for $\alpha=0$ system $(\mathrm{E}(\alpha))$ reduces to Eqs. $(\overline{\mathrm{C}} 1)$ and $(\overline{\mathrm{C}} 2)$, and therefore the outcome associated with every $s \in \operatorname{SFE}_{0}(D, C, N)$ is the Cournot outcome. Hence $\pi(0)=\bar{p}(D, C, N) \bar{q}(D, C, N) \quad C(\bar{q}(D, C, N))$.

Proposition 3.3 establishes the key result mentioned above. Its proof involves tedious calculations, and it is relegated to the appendix. In the proof we use the assumption that the cost function is thrice continuously differentiable and that its third derivative is non negative. As shown in the proof, an implication of this assumption is that SFE are not "very asymmetric", a condition that guarantees the existence of an equilibrium in the class described above that satisfies certain properties. This assumption is also needed in Proposition 3.4 (whose proof appeals to Proposition 3.3), and is satisfied under the conditions imposed in Theorems 3.5 and 3.7 (which proofs appeal to Proposition 3.4).

Proposition 3.3. Let $(D, C, N)$ be an industry such that $C$ is thrice continuously differentiable and satisfies $C^{\prime \prime \prime}(q) \geqslant 0$ for $q>0$. If $\hat{s} \in \operatorname{SFE}(D, C, N)$ is such that $p(\hat{s}) \neq \bar{p}(D, C, N)$, then there is $\alpha>0$ satisfying $\pi(\alpha) \geqslant \max \left\{\pi_{1}(\hat{s}), \ldots, \pi_{n}(\hat{s})\right\}$.

Proposition 3.4 establishes that if the function $\pi$ of an industry $(D, C, N)$ reaches a maximum at $\alpha=0$, then the Cournot outcome is the unique CPSFE outcome. This fact is a straightforward implication of Propositions 3.2 and 3.3.

Proposition 3.4. Let $(D, C, N)$ be an industry such that $C$ is thrice continuously differentiable and satisfies $C^{\prime \prime \prime}(q) \geqslant 0$ for $q>0$. If $\pi(0)>\pi(\alpha)$ for all $\alpha \in(0, \infty)$, then

$$
\left(p(s), q_{1}(s), \ldots, q_{n}(s)\right)=(\bar{p}(D, C, N), \bar{q}(D, C, N), \ldots, \bar{q}(D, C, N))
$$

for all $s \in \operatorname{CPSFE}(D, C, N)$.

Proof. Denote $\bar{p}(D, C, N)=\bar{p}$ and $\bar{q}(D, C, N)=\bar{q}$. Let $s \in \operatorname{SFE}(D, C, N)$ be such that $\left(p(s), q_{1}(s), \ldots, q_{n}(s)\right) \neq(\bar{p}, \bar{q}, \ldots, \bar{q})$. We show that $s \notin C P S F E(D, C, N)$.

Proposition 2.1 implies $p(s) \neq \bar{p}$; hence by Proposition 3.3 there is $\alpha>0$ satisfying $\pi(\alpha) \geqslant \max \left\{\pi_{1}(s), \ldots, \pi_{n}(s)\right\}$. Let $\bar{s} \in \operatorname{CPSFE}(D, C, N)$ be such that $p(\bar{s})=\bar{p}$ and 
$q_{i}(\bar{s})=\bar{q}$ for $i \in N$. (The existence of $\bar{s}$ is guaranteed by Proposition 3.2.) Then we have

$$
\pi_{i}(\bar{s})=\pi(0)>\pi(\alpha) \geqslant \max \left\{\pi_{1}(s), \ldots, \pi_{n}(s)\right\} \geqslant \pi_{i}(s),
$$

for all $i \in N$. Moreover, since $\bar{s} \in \operatorname{CPSFE}(D, C, N), \bar{s}$ is self enforcing. Hence $s \notin \operatorname{CPSFE}(D, C, N)$.

Next, we establish conditions under which in a linear industry the assumptions of Proposition 3.4 hold. A linear industry is described by a linear demand function, (i.e., $D(p)=a \quad b p$, for $p \in\left[0, \frac{a}{b}\right]$, where $a, b \in \mathbb{R}_{+}$), and a linear cost function (i.e., $C(q)=c q$, for $q \geqslant 0$, where $\left.c \in \mathbb{R}_{+}\right)$. Our assumption that $C^{\prime}(0)<\rho$ amounts to assuming $c<\frac{a}{b}$. Thus, a linear industry is described by the parameters $a, b, c$, and $n$. Theorem 3.5 establishes that if there are three or more firms in a linear industry the Cournot outcome is the unique CPSFE outcome. We obtain this result by directly calculating the function $\pi$, and appealing to Proposition 3.4.

Theorem 3.5. Let $(D, C, N)$ be a linear industry. If $n \geqslant 3$, then

$$
\left(p(s), q_{1}(s), \ldots, q_{n}(s)\right)=(\bar{p}(D, C, N), \bar{q}(D, C, N), \ldots, \bar{q}(D, C, N))
$$

for all $s \in \operatorname{CPSFE}(D, C, N)$.

Proof. Let $(D, C, N)$ be a linear industry such that $n \geqslant 3$, and let $a, b$, and $c$ be the parameters describing the demand and cost functions. The solution to system $(\mathrm{E}(\alpha))$ yields

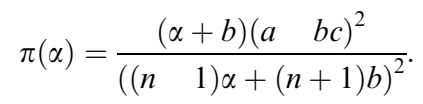

Hence $\pi^{\prime}(\alpha)<0$ on $(0, \infty)$, and therefore $\pi(0)>\pi(\alpha)$ for $\alpha \in(0, \infty)$. Thus, the conclusion of Theorem 3.5 follows from Proposition 3.4.

Note that in an industry where there are only two firms the Stackelberg outcomes are in the Pareto frontier of the set of SFE outcomes, and are therefore CPSFE outcomes. By Theorem 3.5, however, in a linear industry the presence of three or more firms reduces the set of CPSFE outcomes to just the Cournot outcome. Theorem 3.7 below establishes that in fact this conclusion extends to every industry $(D, C, N)$ with a quadratic cost function and linear demand. Nevertheless, as Example 3.6 shows, there are industries with three firms for which outcomes other than the Cournot outcome can be sustained by CPSFE.

Example 3.6. Consider the industry where $D(p)=1 \quad p^{2}$ for $p \in[0,1], C(q)=0$ for $q \in \mathbb{R}$, and $N=\{1,2,3\}$. From the solution to system $(\mathrm{E}(\alpha))$ one can compute the function $\pi$ to obtain

$$
\pi(\alpha)=\frac{1}{49}\left(\sqrt{\left(\alpha^{2}+7\right)} \alpha\right)\left(2+\frac{3 \alpha}{7}\left(\sqrt{ }\left(\alpha^{2}+7\right) \quad \alpha\right)\right) .
$$


This function is uniquely maximized at $\alpha^{*}=\frac{1}{3}$. Let $s \in S F E_{\frac{1}{3}}(D, C, N)$. In this SFE, two of the firms produce $\frac{1}{3}$ and the remaining firm produces $\frac{2}{9}$. We show $s \in \operatorname{CPSFE}(D, C, N)$. Since $\pi\left(\frac{1}{3}\right) \geqslant \pi(\alpha)$ for $\alpha \geqslant 0$, by Proposition $3.3 s$ is Pareto superior to every other SFE outcome. Hence the coalition of all three firms does not have a self enforcing and improving deviation. In addition, the two firms producing $\frac{1}{3}$ are in fact at the Cournot outcome of the "residual industry" (fixing the production of the remaining firm to be $\frac{2}{9}$ ), and therefore by Proposition 3.2 this coalition does not have an improving self enforcing deviation either. Nor does a coalition formed by a firm producing $\frac{1}{3}$ and the firm producing $\frac{2}{9}$ have an improving self enforcing deviation, since these productions correspond to a Stackelberg outcome of their residual industry. Hence $s$ is a CPSFE.

Let $D$ and $C$ be the demand and cost functions of an industry. Denote by $n^{*}$ the smallest integer such that

$$
n^{*} \geqslant 2+\frac{D^{\prime}(\bar{p})+D^{\prime \prime}(\bar{p})\left(\bar{p} \quad C^{\prime}(\bar{q})\right)}{D^{\prime}(\bar{p})\left(1 \quad D^{\prime}(\bar{p}) C^{\prime \prime}(\bar{q})\right)} .
$$

Under appropriate conditions on the functions $D$ and $C, n^{*}$ provides a threshold for the number of firms which guarantees that if $n \geqslant n^{*}$, then the Cournot outcome is the unique outcome that can be sustained by a CPSFE. Note that under our assumptions of demand and cost $n^{*}>2$. Further, if demand is linear, then $n^{*}=3$. Also note that for the industry in Example 3.6 we have

$$
n^{*}=2+\frac{2 \bar{p}+2 \bar{p}}{2 \bar{p}}=4 .
$$

Indeed, in this industry $\pi(0)>\pi(\alpha)$ for $\alpha>0$ whenever there are four or more firms (and therefore by Proposition 3.4 the Cournot outcome is the unique outcome that can be sustained by a CPSFE).

Theorem 3.7 establishes more general conditions than those of Theorem 3.5 under which the Cournot outcome is the unique CPSFE outcome.

Theorem 3.7. Let $(D, C, N)$ be an industry. If

(1) $C^{\prime \prime}(q)=0$ for $q>0, D^{\prime \prime \prime}(p) \leqslant 0$ for $p \in(0, \rho)$, and $n \geqslant n^{*}$, or

(2) $C^{\prime \prime \prime}(q)=0$ for $q>0, D^{\prime \prime}(p)=0$ for $p \in(0, \rho)$, and $n \geqslant 3=n^{*}$,

then

$$
\left(p(s), q_{1}(s), \ldots, q_{n}(s)\right)=(\bar{p}(D, C, N), \bar{q}(D, C, N), \ldots, \bar{q}(D, C, N))
$$

for all $s \in \operatorname{CPSFE}(D, C, N)$.

The following lemma provides alternative conditions that ensure that the function $\pi$ has a unique maximum on $\alpha=0$. These conditions are useful in the proof of Theorem 3.7. The proof of this lemma is given in the appendix. 
Lemma 3.8. Let $(D, C, N)$ be an industry. Assume that for each $\alpha \in(0, \infty)$ either

(1) $n \geqslant n^{*}$ and $\frac{\partial^{2} w}{\partial u^{2}} \geqslant 0$, or

(2) $\left(\begin{array}{llll}n & 1\end{array}\right) \alpha \quad\left(\begin{array}{ll}n & 2\end{array}\right) D^{\prime}(u) \quad \frac{\partial w}{\partial u}>0$

Then $\pi(0)>\pi(\alpha)$ for $\alpha>0$.

With these results in hand we can now prove Theorem 3.7.

Proof of Theorem 3.7. We show that under either of the assumptions of Theorem 3.7 condition (1) of Lemma 3.8 is satisfied, and therefore that $\pi(0)>\pi(\alpha)$ for $\alpha>0$. The conclusion of Theorem 3.7 then follows from Proposition 3.4.

Suppose that (1) of Theorem 3.7 holds. Implicit differentiation of $(E(\alpha) .1)$ yields

$$
\frac{\partial w}{\partial u}=D^{\prime}(u) \quad D^{\prime \prime}(u)\left(u \quad C^{\prime}(w)\right)
$$

and therefore

$$
\frac{\partial^{2} w}{\partial u^{2}}=2 D^{\prime \prime}(u) \quad D^{\prime \prime \prime}(u)\left(u \quad C^{\prime}(w)\right) \geqslant 0 .
$$

Suppose that (2) of Theorem 3.7 holds. Then again differentiating $(\mathrm{E}(\alpha) .1)$ we get

$$
\frac{\partial w}{\partial u}=\frac{D^{\prime}(u)}{1 \quad C^{\prime \prime}(w) D^{\prime}(u)},
$$

and therefore

$$
\frac{\partial^{2} w}{\partial u^{2}}=0 .
$$

Checking conditions (1) and (2) of Lemma 3.8 directly allows us to establish that the Cournot outcome is the unique CPSFE outcome for a quadratic industry (Example 3.9), and for an industry where the cost function is quadratic and the demand is a polynomial of third order (Example 3.10). These examples are outside the scope of Theorem 3.7.

Example 3.9. Consider an industry where $D(p)=1 \quad p^{2}$ for $p \in[0,1], C(q)=\frac{1}{2} q^{2}$ for $q \geqslant 0$, and $N=\{1,2,3\}$. For this industry, the Cournot outcome is the unique outcome that can be sustained by a CPSFE. We show that (1) of Lemma 3.8 holds; hence that $\pi^{\prime}(\alpha)<0$ for $\alpha>0$, and therefore this result follows from Proposition 3.4.

Let $\alpha>0$. From $(\mathrm{E}(\alpha) .1)$ we have

$$
w=2 u(u \quad w)=2 \frac{u^{2}}{1+2 u} .
$$

Hence

$$
\frac{\partial^{2} w}{\partial u^{2}}=\frac{4}{(1+2 u)^{3}}>0 .
$$


Example 3.10. Consider an industry where $D(p)=\frac{1}{3}\left(27 \quad p^{3}\right)$, for $p \in[0,3], C(q)=$ $\frac{1}{2} q^{2}$ for $q \geqslant 0$, and $N=\{1,2,3\}$. The Cournot outcome for this industry is $\bar{p}=$ 2.1977, $\bar{q}=1.8206$. Implicit differentiation of $(\mathrm{E}(\alpha) .1)$ yields (see the appendix)

$$
\frac{\partial^{2} w}{\partial u^{2}}(\bar{p})=\frac{2 \bar{p}\left(3 \quad \bar{p}^{2}\right)}{\left(1+\bar{p}^{2}\right)^{3}}<0,
$$

hence (1) of Lemma 3.8 does not hold. Nonetheless, it is easy to prove that (2) of Theorem 3.7 holds. Write

$$
\gamma(\alpha)=\frac{1}{n} 1\left(\begin{array}{llll}
n & 1) \alpha & \left(\begin{array}{ll}
n & 2
\end{array}\right) D^{\prime}(u) & \frac{\partial w}{\partial u}
\end{array}\right)=\frac{1}{2}\left(\begin{array}{lll}
2 \alpha & D^{\prime}(u) & \frac{\partial w}{\partial u}
\end{array}\right) .
$$

We show $\gamma(\alpha) \leqslant 0$. One can compute $\gamma(0)$ (see the appendix) to obtain

$$
\gamma(0)=D^{\prime}(\bar{p})\left(\begin{array}{ll}
n & n^{*}
\end{array}\right)
$$

As

$$
n=3>n^{*}=2+\frac{\bar{p}^{2}+2 \bar{p}(\bar{p} \quad \bar{q})}{\bar{p}^{2}\left(1+\bar{p}^{2}\right)}=2.2304
$$

we have $\gamma(0)<0$. Hence it suffices to show that $\gamma^{\prime}(\alpha)<0$. We have

$$
\gamma^{\prime}(\alpha)=\left(1 \quad D^{\prime \prime}(u) u^{\prime}(\alpha)\right)+\frac{1}{n \quad 1}\left(D^{\prime \prime}(u)+\frac{\partial^{2} w}{\partial u^{2}}\right) u^{\prime}(\alpha),
$$

and since $u^{\prime}(\alpha)<0$, then $D^{\prime \prime}(u)+\frac{\partial^{2} w}{\partial u^{2}}>0$ implies $\gamma^{\prime}(\alpha)<0$. Here we have

$$
D^{\prime \prime}(u)+\frac{\partial^{2} w}{\partial u^{2}}=2 u\left(1+\frac{3 \quad u^{2}}{\left(1+u^{2}\right)^{3}}\right)>0 .
$$

\subsection{Uniqueness and cost asymmetries}

In this section we discuss the robustness of the uniqueness result in the context of a simple linear asymmetric industry with three firms. We show that if marginal costs are sufficiently asymmetric there are multiple CPSFE outcomes. We also show that when firms' costs are nearly symmetric, the Pareto superiority of the Cournot outcome is preserved. Hence the result that the Cournot outcome is the unique CPSFE outcome is robust to cost asymmetries. (Actually, we prove that the Cournot outcome is Pareto superior to every outcome generated by a SFE in the class $\operatorname{SFE}_{\alpha}\left(D, C_{1}, C_{2}, C_{3},\{1,2,3\}\right)$ with $\alpha>0$, described in Section 3.3. Establishing uniqueness of CPSFE formally requires showing that Proposition 3.3 holds for this industry if costs are nearly symmetric. The proof of this result is cumbersome, and we omit it.)

Consider a linear industry where there are three firms whose cost functions are $C_{1}(q)=0$, and $C_{2}(q)=C_{3}(q)=c q$, with $0 \leqslant c \leqslant \frac{1}{3}$. The demand is given, without loss of generality, by $D(p)=1 p$. Consider the SFE equilibrium $\hat{s} \in \operatorname{SFE}_{1}\left(D, C_{1}, C_{2}, C_{3}, N\right)$, given by $\hat{s}_{1}(p)=\hat{q}_{1}=\frac{1}{3}+c, \hat{s}_{2}(p)=\hat{q}_{2}=\frac{1}{3} \quad c$, and $\hat{s}_{3}$ 


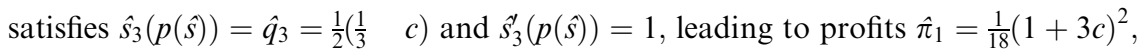
and $\hat{\pi}_{2}=2 \hat{\pi}_{3}=\frac{1}{18}\left(\begin{array}{ll}1 & 3 c\end{array}\right)^{2}$. (It is easy to check that $\left(\hat{p}, \hat{q}_{1}, \hat{q}_{2}, \hat{q}_{3}, \hat{\alpha}\right)$, with $\hat{p}=$ $\frac{1}{6}(1+3 c)$ and $\hat{\alpha}=1$ are a solution to system $(\mathrm{E}(\alpha))$, suitably modified for this industry with cost asymmetries this system includes four rather than three equations; see below.) This equilibrium can be interpreted as one where firm 3 behaves as a Stackelberg follower, and firms 1 and 2, the leaders, compete á la Cournot.

At the Cournot outcome of this industry, profits are $\bar{\pi}_{1}=\frac{1}{16}(1+2 c)^{2}$ and $\bar{\pi}_{2}=$ $\bar{\pi}_{3}=\frac{1}{16}\left(\begin{array}{ll}1 & 2 c\end{array}\right)^{2}$. Thus, $\hat{\pi}_{1} \quad \bar{\pi}_{1}=\frac{1}{144}\left(12 c+36 c^{2} \quad 1\right)$. Hence for some parameter values, e.g., $c=\frac{1}{6}$, we have $\hat{\pi}_{1} \quad \bar{\pi}_{1}>0$; i.e., the Cournot outcome is not Pareto superior to the outcome generated by the SFE $\hat{s}$ described above. Indeed, it is easy to see that if $c=\frac{1}{6}$ the profile $\hat{s}$ is a CPSFE this can be shown by an argument similar to that used in Example 3.6 above.

For values of $c$ near zero, however, the Cournot outcome is Pareto superior to the outcome generated by $\hat{s}$ (i.e., $\bar{\pi}_{i}>\hat{\pi}_{i}$ for $i \in N$ ). Moreover, for values of $c$ near zero the Cournot outcome is Pareto superior to all the outcomes generated by SFE in the class $\operatorname{SFE}_{\alpha}\left(D, C_{1}, C_{2}, C_{3}, N\right)$ with $\alpha>0$. To see this, note that in this industry with cost asymmetries we must consider two types of SFE in the class $\operatorname{SFE}_{\alpha}\left(D, C_{1}, C_{2}, C_{3}, N\right)$, namely,

(a) those in which firms 1 and 2 (or 1 and 3) act as leaders, competing á la Cournot, and firm 3 (2) behaves as a follower, and

(b) those where firms 2 and 3 act as leaders, competing á la Cournot, and firm 1 behaves as a follower.

For each type of SFE, (a) and (b), by solving the appropriate version of system of equations $(\mathrm{E}(\alpha))$ we can calculate the profits of the leaders (as a function of $\alpha$, the slope of the followers supply) and $c$ (the marginal cost of firms 1 and 2). For the SFE of type (a) system $\left(E^{a}(\alpha)\right)$ is

$$
\begin{aligned}
& q_{1}=(1+\alpha) p, \\
& q_{2}=(1+\alpha)(p \quad c), \\
& q_{3}=\left(\begin{array}{ll}
p & c
\end{array}\right), \\
& q_{1}+q_{2}+q_{3}=1 \quad p .
\end{aligned}
$$

At these equilibria the profits of the leaders, say firms 1 and 2, are

$$
\pi_{1}^{\mathrm{a}}(\alpha, c)=\frac{1}{4}(1+c(2+\alpha))^{2} \frac{1+\alpha}{(2+\alpha)^{2}}
$$

and

$$
\pi_{2}^{\mathrm{a}}(\alpha, c)=\frac{1}{4}(1 \quad 2 c+\alpha c)(1 \quad c(2+\alpha)) \frac{1+\alpha}{(2+\alpha)^{2}} .
$$

For values of $c$ near zero both these functions are decreasing in $\alpha$, and therefore the Cournot outcome is Pareto superior to the outcomes generated by these equilibria. 
For the SFE of type (b), solving system $\left(\mathrm{E}^{\mathrm{b}}(\alpha)\right)$ we calculate the profits of the leaders,

$$
\pi_{2}^{\mathrm{b}}(\alpha, c)=\pi_{3}^{\mathrm{b}}(\alpha, c)=\frac{1}{4}\left(\begin{array}{ll}
2 c & 1)^{2} \\
\frac{1+\alpha}{(2+\alpha)^{2}}
\end{array}\right.
$$

Again for values of $c$ near zero this function is decreasing in $\alpha$, and therefore the Cournot outcome is Pareto superior to the outcomes generated by these equilibria.

\section{Acknowledgments}

We benefited from the comments and suggestions of an associate editor and two referees, as well as those of John Wooders and the participants at seminars at the University of Arizona, Arizona State University, Brown University, and CORE, and at the Young Economists Meeting in Amsterdam and the Summer School of the European Economic Association in Toulouse. Financial support from the Comisión Nacional del Sistema Eléctrico, and the Spanish Ministry of Education, Grant SEC2001 0973, is gratefully acknowledged. The opinions expressed in this paper are the sole responsibility of the authors, and are not those of the European Commission.

\section{Appendix}

Proof of Proposition 3.3. Let $(D, C, N)$ be an industry such that $C^{\prime \prime \prime}(q) \geqslant 0$ for $q>0$ and let $\hat{s} \in \operatorname{SFE}(D, C, N)$. Write $\bar{p}(D, C, N)=\bar{p}, \bar{q}(D, C, N)=\bar{q}, p(\hat{s})=\hat{p}$, and $q_{i}(\hat{s})=\hat{q}_{i}, \hat{\alpha}_{i}=\hat{s}_{i}^{\prime}(\hat{p})$, and $\hat{A}_{i}=\sum_{j \in N \backslash\{i\}} \hat{\alpha}_{j}$ for $i \in N$. Assume that $\hat{p} \neq \bar{p}$.

Without loss of generality assume that $\hat{q}_{1} \geqslant \hat{q}_{2} \geqslant \cdots \geqslant \hat{q}_{n}$; thus

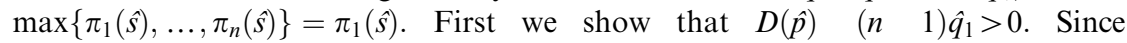
$\hat{s} \in \operatorname{SFE}(D, C, N)$, for $i \in N$ we have

$$
\hat{q}_{i}=\left(\hat{A}_{i} \quad D^{\prime}(\hat{p})\right)\left(\hat{p} \quad C^{\prime}\left(\hat{q}_{i}\right)\right),
$$

by condition $\left(\mathrm{E}_{i}\right)$. Hence for $i \in N$ we have

$$
\begin{aligned}
& \hat{q}_{i}=\hat{q}_{1} \quad\left(\hat{A}_{1} \quad D^{\prime}(\hat{p})\right)\left(\hat{p} \quad C^{\prime}\left(\hat{q}_{1}\right)\right)+\left(\begin{array}{lll}
\hat{A}_{i} & \left.D^{\prime}(\hat{p})\right)\left(\hat{p} \quad C^{\prime}\left(\hat{q}_{i}\right)\right)
\end{array}\right.
\end{aligned}
$$

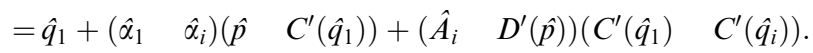

Thus, since $\hat{p}$ maximizes the profits of firm 1 on the residual demand, we have $\hat{p} \geqslant C^{\prime}\left(q_{1}\right)$, and therefore

$$
D(\hat{p}) \quad\left(\begin{array}{ll}
n & 1
\end{array}\right) \hat{q}_{1}=\left(\hat{q}_{1}+\sum_{i>1} \hat{q}_{i}\right) \quad\left(\begin{array}{ll}
n & 1
\end{array}\right) \hat{q}_{1}
$$




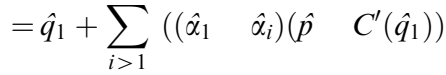

$$
\begin{aligned}
& +\left(\begin{array}{ll}
\hat{A}_{i} & \left.D^{\prime}(\hat{p})\right)\left(C^{\prime}\left(\hat{q}_{1}\right) \quad C^{\prime}\left(\hat{q}_{i}\right)\right)
\end{array}\right) \\
& =\left(\begin{array}{lll}
\hat{A}_{1} & D^{\prime}(\hat{p})+\left(\begin{array}{lll}
n & 1
\end{array}\right) \hat{\alpha}_{1} & \hat{A}_{1}
\end{array}\right)\left(\begin{array}{ll}
\hat{p} & C^{\prime}\left(\hat{q}_{1}\right)
\end{array}\right) \\
& +\sum_{i>1}\left(\hat{A}_{i} \quad D^{\prime}(\hat{p})\right)\left(C^{\prime}\left(\hat{q}_{1}\right) \quad C^{\prime}\left(\hat{q}_{i}\right)\right) \\
& =\left(\left(\begin{array}{lll}
n & 1) \hat{\alpha}_{1} & \left.D^{\prime}(\hat{p})\right)\left(\hat{p} \quad C^{\prime}\left(\hat{q}_{1}\right)\right)
\end{array}\right.\right. \\
& +\sum_{i>1}\left(\hat{A}_{i} \quad D^{\prime}(\hat{p})\right)\left(C^{\prime}\left(\hat{q}_{1}\right) \quad C^{\prime}\left(\hat{q}_{i}\right)\right)>0 .
\end{aligned}
$$

Let $\check{p}$ be such that $D(\tilde{p}) \quad(n \quad 1) \hat{q}_{1}=0$, and for $u \in[\hat{p}, \check{p}]$ define

$$
\left.\left.\phi(u)=D(u) \quad(n \quad 1) \hat{q}_{1}+D^{\prime}(u)\left(\begin{array}{llll}
u & C^{\prime}(D(u) & (n & 1
\end{array}\right) \hat{q}_{1}\right)\right) .
$$

We establish below that the equation

$$
\phi(u)=0
$$

has a unique solution on $[\hat{p}, \tilde{p}]$, $\tilde{u}$. Hence $(\tilde{u}, \tilde{w}, \tilde{\alpha})$, where

$$
\tilde{w}=D(\tilde{u}) \quad(n \quad 1) \hat{q}_{1}
$$

and

$$
\tilde{\alpha}=D^{\prime}(\tilde{u})+\frac{\hat{q}_{1}}{\tilde{u} \quad C^{\prime}\left(\hat{q}_{1}\right)}
$$

is the unique solution to the system $E(\alpha)$ for $v^{*}=\hat{q}_{1}$. Moreover, $\tilde{u} \geqslant \hat{p}$ (because $\phi(\hat{p}) \geqslant 0$ and $\left.\phi^{\prime}<0\right)$. Further $\tilde{w}>0$, and $\tilde{\alpha} \geqslant 0$. To see this note that $\tilde{u} \geqslant \hat{p}$ and $\hat{q}_{1} \geqslant \hat{q}_{i}$ for $i>1$ imply

$$
\begin{aligned}
\hat{q}_{n} & =D(\hat{p}) \quad \hat{q}_{1} \quad \sum_{i=2}^{n-1} \hat{q}_{i} \\
& \geqslant D(\tilde{u}) \quad(n \quad 1) \hat{q}_{1}=\tilde{w} .
\end{aligned}
$$

And since $C$ is increasing, we have $\tilde{u} \quad C^{\prime}(\tilde{w}) \geqslant \hat{p} \quad C^{\prime}\left(\hat{q}_{n}\right) \geqslant 0$, and therefore

$$
\tilde{w}=D^{\prime}(\tilde{u})\left(\tilde{u} \quad C^{\prime}(\tilde{w})\right)>0 .
$$

Now, because $C^{\prime}$ is increasing, $\hat{q}_{1} \geqslant \hat{q}_{n} \geqslant \tilde{w}$ implies

$$
\tilde{\alpha}=D^{\prime}(\tilde{u})+\frac{\hat{q}_{1}}{\tilde{u} \quad C^{\prime}\left(\hat{q}_{1}\right)}=\frac{\hat{q}_{1}}{\tilde{u} \quad C^{\prime}\left(\hat{q}_{1}\right)} \quad \frac{\tilde{w}}{\tilde{u} \quad C^{\prime}(\tilde{w})} \geqslant \frac{\hat{q}_{1} \quad \tilde{w}}{\tilde{u} \quad C^{\prime}(\tilde{w})} \geqslant 0 .
$$

Moreover, it is easy to see that if $\hat{q}_{1}=\hat{q}_{n}=\tilde{w}$ then $\hat{q}_{1}=\cdots=\hat{q}_{n}=\bar{q}$ and $\hat{p}=\tilde{u}=\bar{p}$, which contradicts that $\hat{p} \neq \bar{p}$. Thus $\hat{q}_{1}>\tilde{w}$ and therefore $\tilde{\alpha}>0$.

Let $s$ be a profile of supply functions satisfying for $i \in N \backslash\{n\}, \tilde{s}_{i}(p)=\hat{q}_{1}$ for $p \in[0, \rho]$, and $\tilde{s_{n}}(\tilde{u})=\tilde{w}, \tilde{s}_{n}^{\prime}(\tilde{u})=\tilde{\alpha}$. The above construction implies $\tilde{s} \in \operatorname{SFE}_{\tilde{\alpha}}(D, C, N)$. 
Moreover, $p(\tilde{s})=\tilde{u}=u(\tilde{\alpha}) \geqslant \hat{p}=p(\hat{s})$, and

$$
\pi_{1}(\tilde{s})=p(\tilde{s}) \tilde{s}_{1}(p(\tilde{s})) \quad C\left(\tilde{s}_{1}(p(\tilde{s}))\right)=p(\tilde{s}) \hat{q}_{1} \quad C\left(\hat{q}_{1}\right) \geqslant \hat{p} \hat{q}_{1} \quad C\left(\hat{q}_{1}\right)=\pi_{1}(\hat{s}),
$$

which establishes the conclusion of Proposition 3.3.

It remains to be shown that $\phi(u)=0$ has a unique solution on $[\hat{p}, \check{p}]$. Since $\check{p}>\hat{p}$, $0<\hat{p} \quad C^{\prime}\left(\hat{q}_{1}\right)<\check{p} \quad C^{\prime}(0)$, and therefore

$$
\begin{aligned}
\phi(\check{p}) & =D(\check{p}) \quad\left(\begin{array}{ll}
n & 1
\end{array}\right) \hat{q}_{1}+D^{\prime}(\check{p})\left(\check{p} \quad C^{\prime}\left(D(\check{p}) \quad\left(\begin{array}{ll}
n & 1
\end{array}\right) \hat{q}_{1}\right)\right) \\
& =D^{\prime}(\check{p})\left(\check{p} \quad C^{\prime}(0)\right)<0 .
\end{aligned}
$$

We show that $\phi(\hat{p}) \geqslant 0$. Denote $\hat{q}=D(\hat{p}) \quad\left(\begin{array}{ll}n & 1\end{array}\right) \hat{q}_{1}$. Then we have

$$
\begin{aligned}
& \phi(\hat{p})=D(\hat{p}) \quad\left(\begin{array}{ll}
n & 1
\end{array}\right) \hat{q}_{1}+D^{\prime}(\hat{p})\left(\hat{p} \quad C^{\prime}(\hat{q})\right)
\end{aligned}
$$

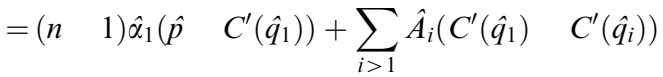

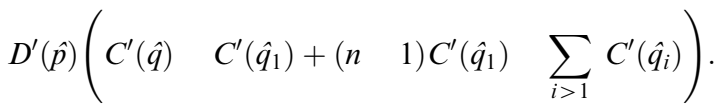

As noted above $\hat{p} \geqslant C^{\prime}\left(\hat{q}_{1}\right)$; moreover, because $C$ is a convex function and $\hat{q}_{1} \geqslant \hat{q}_{i}$, we have $C^{\prime}\left(\hat{q}_{1}\right) \geqslant C^{\prime}\left(\hat{q}_{i}\right)$ for $i>1$. Hence the first term in the above expression is non negative; i.e.,

$$
\left(\begin{array}{ll}
n & 1)
\end{array}\right) \hat{\alpha}_{1}\left(\hat{p} \quad C^{\prime}\left(\hat{q}_{1}\right)\right)+\sum_{i>1} \hat{A}_{i}\left(C^{\prime}\left(\hat{q}_{1}\right) \quad C^{\prime}\left(\hat{q}_{i}\right)\right) \geqslant 0 .
$$

We show that the second term is also non negative. Since $\hat{q}_{1} \geqslant \hat{q}_{i}$ for $i>1$, we have $\hat{q}_{i}=D(\hat{p}) \quad \sum_{j \neq i} \hat{q}_{j} \geqslant D(\hat{p}) \quad(n \quad 1) \hat{q}_{1}=\hat{q}$ for $i>1$. Thus, for $i>1$ there is $\lambda_{i} \in[0,1]$ such that $\hat{q}_{i}=\lambda_{i} \hat{q}_{1}+\left(\begin{array}{ll}1 & \lambda_{i}\end{array}\right) \hat{q}$. Hence

$$
\hat{q}+\left(\begin{array}{ll}
n & 1
\end{array}\right) \hat{q}_{1}=D(\hat{p})=\hat{q}_{1}+\sum_{i>1} \hat{q}_{i}=\hat{q}_{1}+\sum_{i>1}\left(\lambda_{i} \hat{q}_{1}+\left(\begin{array}{ll}
1 & \lambda_{i}
\end{array}\right) \hat{q}\right) .
$$

Thus,

$$
\hat{q}_{1} \quad \hat{q}=\left(\begin{array}{ll}
\hat{q}_{1} & \hat{q}
\end{array}\right) \sum_{i>1}\left(\begin{array}{ll}
1 & \lambda_{i}
\end{array}\right),
$$

and therefore either $\sum_{i>1}\left(\begin{array}{ll}1 & \lambda_{i}\end{array}\right)=1$ or $\hat{q}_{1}=\hat{q}$. This equation together with our assumption that $C^{\prime \prime \prime}(q) \geqslant 0$ for $q>0$, yields the inequality

$$
\begin{aligned}
& \left(\begin{array}{ll}
n & 1
\end{array}\right) C^{\prime}\left(\hat{q}_{1}\right) \quad \sum_{i>1} C^{\prime}\left(\hat{q}_{i}\right)=\left(\begin{array}{ll}
n & 1
\end{array}\right) C^{\prime}\left(\hat{q}_{1}\right) \quad \sum_{i>1} C^{\prime}\left(\lambda_{i} \hat{q}_{1}+\left(\begin{array}{ll}
1 & \lambda_{i}
\end{array}\right) \hat{q}\right) \\
& \geqslant\left(\begin{array}{ll}
n & 1
\end{array}\right) C^{\prime}\left(\hat{q}_{1}\right) \quad \sum_{i>1} \lambda_{i} C^{\prime}\left(\hat{q}_{1}\right)+\sum_{i>1}\left(\begin{array}{ll}
1 & \lambda_{i}
\end{array}\right) C^{\prime}(\hat{q})
\end{aligned}
$$

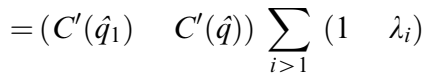

$$
\begin{aligned}
& =C^{\prime}\left(\hat{q}_{1}\right) \quad C^{\prime}(\hat{q}) \text {. }
\end{aligned}
$$


Hence

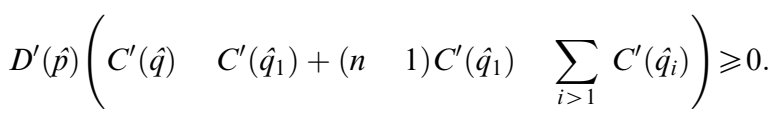

Therefore $\phi(\hat{p}) \geqslant 0$.

The Intermediate Value Theorem implies that the equation $\phi(u)=0$ has a solution on $[\hat{p}, \tilde{p}]$. Moreover, the solution is unique since $\phi^{\prime}<0$.

Proof of Lemma 3.8. Let $\alpha>0$. From the definition of $\pi$ we have

$$
\pi^{\prime}(\alpha)=u^{\prime}(\alpha) v(\alpha)+\left(u(\alpha) \quad C^{\prime}(v)\right)\left(\frac{\partial v}{\partial u} u^{\prime}(\alpha)+\frac{\partial v}{\partial \alpha}\right) .
$$

From $(\mathrm{E}(\alpha) .1)$ we have

$$
\frac{\partial w}{\partial u}=\frac{D^{\prime}(u) \quad D^{\prime \prime}(u)\left(u \quad C^{\prime}(w)\right)}{1 \quad C^{\prime \prime}(w) D^{\prime}(u)}>0 .
$$

From $(\mathrm{E}(\alpha) .2)$ we get

$$
\frac{\partial v}{\partial \alpha}=\frac{\left(u \quad C^{\prime}(v)\right)}{1+C^{\prime \prime}(v)\left(\alpha \quad D^{\prime}(u)\right)}>0
$$

and

$$
\frac{\partial v}{\partial u}=\frac{\alpha \quad D^{\prime}(u) \quad D^{\prime \prime}(u)\left(u \quad C^{\prime}(v)\right)}{1+C^{\prime \prime}(v)\left(\alpha \quad D^{\prime}(u)\right)}>0 .
$$

From $(\mathrm{E}(\alpha) .3)$ we have

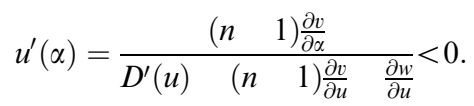

Substituting in the expression of $\pi^{\prime}(\alpha)$ we have

$$
\begin{aligned}
& \pi^{\prime}(\alpha)=u^{\prime}(\alpha)\left(v(\alpha)+(u(\alpha)) \quad C^{\prime}(v(\alpha)) \frac{1}{n \quad 1}\left(\begin{array}{ll}
D^{\prime}(u(\alpha)) & \left.\left.\frac{\partial w}{\partial u}\right)\right)
\end{array}\right.\right.
\end{aligned}
$$

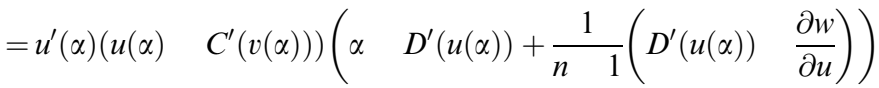

$$
\begin{aligned}
& =\beta(\alpha) \gamma(\alpha),
\end{aligned}
$$

where

$$
\beta(\alpha)=u^{\prime}(\alpha)\left(u(\alpha) \quad C^{\prime}(v(\alpha))\right)>0,
$$

and

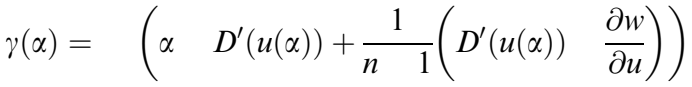

$$
\begin{aligned}
& \left.=\frac{1}{n} 1\left(\begin{array}{llll}
(n & 1
\end{array}\right) \alpha \quad\left(\begin{array}{ll}
n & 2
\end{array}\right) D^{\prime}(u(\alpha)) \quad \frac{\partial w}{\partial u}\right) .
\end{aligned}
$$

This establishes the conclusion of Lemma 3.8 under (2). 
Note that

$$
\begin{aligned}
&\left(\begin{array}{lll}
n \quad 1) \gamma(0) & =\left(\begin{array}{lll}
n & 2
\end{array}\right) D^{\prime}(\bar{p}) & \frac{D^{\prime}(\bar{p})+D^{\prime \prime}(\bar{p})(\bar{p}}{1} C^{\prime}(\bar{q})
\end{array}\right) \\
&=D^{\prime \prime}(\bar{p})\left(\begin{array}{llll}
n & 2 & \frac{D^{\prime}(\bar{p})+D^{\prime}(\bar{p})}{D^{\prime \prime}(\bar{p})(\bar{p}} & \left.C^{\prime}(\bar{q})\right)
\end{array}\right) \\
&=D^{\prime}(\bar{p})\left(\begin{array}{lll}
n & \left.n^{*}\right) \leqslant 0 .
\end{array}\right.
\end{aligned}
$$

We establish Lemma 3.8 under (1) by showing that $\gamma^{\prime}(\alpha)<0$ for $\alpha \in(0, \infty)$. We have

$$
\begin{aligned}
\gamma^{\prime}(\alpha) & \left.=\frac{1}{n} 1\left(\begin{array}{ll}
n & 1
\end{array}\right)+\left(\begin{array}{ll}
n & 2
\end{array}\right) D^{\prime \prime}(u) u^{\prime}(\alpha)+\frac{\partial^{2} w}{\partial u^{2}} u^{\prime}(\alpha)\right) \\
& =\quad\left(\begin{array}{ll}
1 & \left.D^{\prime \prime}(u) u^{\prime}(\alpha)\right)+\frac{1}{n} 1
\end{array}\left(D^{\prime \prime}(u)+\frac{\partial^{2} w}{\partial u^{2}}\right) u^{\prime}(\alpha) .\right.
\end{aligned}
$$

Since $\frac{\partial^{2} w}{\partial u^{2}} \geqslant 0$ and $u^{\prime}(\alpha)<0$, we have (recall that $D$ is a concave function)

$$
\left(D^{\prime \prime}(u)+\frac{\partial^{2} w}{\partial u^{2}}\right) u^{\prime}(\alpha) \leqslant 0
$$

Hence in order to prove that $\gamma^{\prime}(\alpha)<0$ it suffices to show that $1 \quad D^{\prime \prime}(u) u^{\prime}(\alpha)>0$. As $u^{\prime}(\alpha)<0,(\mathrm{E}(\alpha) .3)$ implies

$$
0<D^{\prime}(u) u^{\prime}(\alpha)=\left(\begin{array}{ll}
n & 1
\end{array}\right)\left(\frac{\partial v}{\partial u} u^{\prime}(\alpha)+v^{\prime}(\alpha)\right)+\frac{\partial w}{\partial u} u^{\prime}(\alpha) .
$$

Since

$$
\frac{\partial w}{\partial u} u^{\prime}(\alpha)<0
$$

the above inequality implies

$$
\frac{\partial v}{\partial u} u^{\prime}(\alpha)+v^{\prime}(\alpha)>0
$$

Since

$$
v^{\prime}(\alpha)=\frac{\left(u \quad C^{\prime}(v)\right)}{1+C^{\prime \prime}(v)\left(\alpha \quad D^{\prime}(u)\right)}>0,
$$

we have

$$
\begin{aligned}
\frac{\partial v}{\partial u} u^{\prime}(\alpha)+v^{\prime}(\alpha) & =\left(\begin{array}{lll}
\alpha & D^{\prime}(u) D^{\prime \prime}(u)\left(u \quad C^{\prime}(v)\right) \\
1+C^{\prime \prime}(v)(\alpha & \left.D^{\prime}(u)\right)
\end{array}\right) u^{\prime}(\alpha)+v^{\prime}(\alpha) \\
& =\left(\frac{\alpha}{1+C^{\prime \prime}(v)\left(\alpha \quad D^{\prime}(u)\right)}\right) u^{\prime}(\alpha)+\left(\begin{array}{ll}
1 & \left.D^{\prime \prime}(u) u^{\prime}(\alpha)\right) v^{\prime}(\alpha)>0
\end{array}\right.
\end{aligned}
$$

The first term in the right hand side of the above expression is negative. Hence the second term must be positive. Since $v^{\prime}(\alpha)>0$, we must have $1 \quad D^{\prime \prime}(u) u^{\prime}(\alpha)>0$. Therefore $\gamma^{\prime}(\alpha)<0$, and since $\gamma(0) \leqslant 0$, we have $\gamma(\alpha)<0$ for $\alpha>0$. 


\section{References}

[1] R. Aumann, Acceptable points in general cooperative n-person games, in: H.W. Kuhn, A.W. Tucker (Eds.), Contributions to the Theory of Games IV, Princeton University Press, Princeton, NJ, 1959.

[2] R. Amir, V. Lambson, On the effects of entry in Cournot markets, Rev. Econom. Stud. 67 (2000) 235-254.

[3] B. Bernheim, B. Peleg, M. Whinston, Coalition-proof Nash equilibria: I concepts, J. Econom. Theory 42 (1987) 1-12.

[4] B. Bernheim, M. Whinston, Coalition-proof Nash equilibria: II applications, J. Econom. Theory 42 (1987) 13-29.

[5] Boletín Oficial del Estado (Spain), Reglas del Mercado de Producción de Energía Eléctrica, May 20, 2001 (English translation: http://www.comel.es/es/reglas_contrato/mreglasconadhesionfr.htm).

[6] R. Green, D. Newbery, Competition in the British electricity spot market, J. Polit. Econom. 100 (1992) 929-953.

[7] S. Grossman, Nash equilibrium and the industrial organization of markets with large fixed costs, Econometrica 49 (1981) 1149-1172.

[8] O. Hart, Imperfect competition in general equilibrium: an overview of recent work, in: K. Arrow, S. Honkaphoja (Eds.), Frontiers in Econometrics, Basil Blackwell, Oxford, 1985.

[9] P. Klemperer, M. Meyer, Price competition vs. quantity competition: the role of uncertainty, Rand J. Econom. 17-4 (1986) 618-638.

[10] P. Klemperer, M. Meyer, Supply function equilibria in oligopoly under uncertainty, Econometrica 57 (1989) 1243-1277.

[11] D. Kreps, J. Scheinkman, Quantity precommitment and Bertrand competition yield Cournot outcomes, Bell J. Econom. 14 (1983) 326-337.

[12] D. Moreno, J. Wooders, Coalition-proof equilibrium, Games Econom. Behavior 17 (1996) 80-112.

[13] S.-S. Yi, On the coalition-proofness of the Pareto frontier of the set of Nash equilibria, Games Econom. Behavior 26 (1999) 353-364. 